\title{
Fraturas em duas e três partes do úmero proximal tratadas com sutura não absorvível ${ }^{*}$
}

\author{
Two- and three-part fractures of the proximal \\ humerus treated with non-absorbable suture
}

Marco Antônio de Castro Veado ${ }^{1}$, Adher Leonardo Leite Moura ${ }^{2}$

\section{RESUMO}

Objetivo: Avaliar os resultados clínicos e radiográficos dos pacientes com fraturas em duas e três partes da extremidade proximal do úmero tratados por sutura não absorvível, com incorporação do manguito rotador, o que permite aumentar a estabilidade da fixação, principalmente em pacientes com pobre qualidade óssea. Métodos: Dezenove pacientes foram operados, sendo 15 do sexo feminino e quatro do masculino, com média de idade de 57,4 anos (23-79 anos) e seguimento médio de 53,4 meses (sete a 144 meses). De acordo com a classificação de Neer, havia 10 fraturas em três partes (colo cirúrgico e tubérculo maior) e nove fraturas em duas partes (duas do tubérculo maior e sete do colo cirúrgico). Todos foram operados na posição "cadeira de praia", com anestesia endotraqueal e blo-

* Trabalho realizado no Hospital Governador Israel Pinheiro, Hospital Mater Dei e Faculdade de Ciências Médicas, Hospital Universitário São José. Belo Horizonte (MG), Brasil.

1. Professor Assistente da Faculdade de Ciências Médicas de Minas Gerais; Cirurgião do Grupo de Ombro do Hospital Mater Dei e Hospital Governador Israel Pinheiro, Belo Horizonte (MG), Brasil.

2. Membro da Sociedade Brasileira de Cirurgia do Ombro e Cotovelo. Indicar a instituição que o autor pertence.

Endereço para correspondência: Rua Celso Porfírio Machado, 104, Belvedere - 303204-000 - Belo Horizonte (MG), Brasil. Tel.: (31) 3286-3156.

E-mail: castrove@terra.com.br

Recebido em 22/2/07. Aprovado para publicação em 16/10/07. Copyright RBO2007 queio do plexo braquial. $O$ acesso deltopeitoral foi utilizado para fraturas em duas partes do colo cirúrgico e fraturas em três partes. Para fraturas em duas partes do tubérculo maior, foi utilizado acesso transacromial. As fraturas foram reduzidas e fixadas com sutura não absorvível no 5 . O ângulo cervicodiafisário foi medido para avaliação da consolidação viciosa. Resultados: Verificou-se consolidação óssea em $95 \%$ dos pacientes. A média da elevação anterior nos pacientes com fratura em duas partes foi de $163,3^{\circ}\left(90^{\circ}-180^{\circ}\right)$; a rotação externa média, de $56^{\circ}\left(30^{\circ}\right.$ a $\left.90^{\circ}\right)$; e a rotação interna média, de T10 (S1-T5). Nos pacientes com fratura em três partes, a média de elevação anterior foi de $163^{\circ}\left(100^{\circ}-180^{\circ}\right)$; a rotação externa média, de $52,5^{\circ}$ $\left(5^{\circ}-70^{\circ}\right)$ e a rotação interna média, de T10 (L4-T7). A força de abdução foi igual à do lado contralateral em 12 pacientes e menor em sete. $O$ tempo necessário para o paciente retornar às atividades anteriores foi, em média, de 5,19 meses (dois a 12 meses). Na avaliação radiológica, o ângulo cervicodiafisário médio foi de $141^{\circ}\left(110^{\circ}-170^{\circ}\right)$ nas fraturas em duas partes e de $150^{\circ}\left(106^{\circ}-210^{\circ}\right)$ nas fraturas em três partes, sendo verificada consolidação em valgo em nove pacientes (59\%) e, em varo, em dois pacientes. De acordo com o escore da UCLA, verificaram-se $88,8 \%$ de bons e excelentes resultados e $11,2 \%$ de maus resultados, para ambos os tipos de fratura. Os últimos, representados por um caso de pseudartrose e outro, por uma capsulite adesiva no pós-operatório. Conclusão: As fraturas em duas e três partes do úmero proximal podem ser 
satisfatoriamente tratadas com sutura não absorvível com incorporação do manguito rotador, particularmente nos idosos. Complicações relacionadas aos metais estão afastadas. Pacientes com fraturas do tubérculo maior tratados com suturas podem ter resultados similares aos daqueles com fraturas do colo cirúrgico. Embora o objetivo da cirurgia seja a reconstrução anatômica, alguma deformidade residual não impede resultado satisfatório. O método é pouco invasivo, permite estabilidade razoável dos fragmentos, com altos índices de consolidação óssea e satisfação do paciente. A cooperação do paciente é crucial para o sucesso terapêutico.

Descritores - Fraturas do úmero/radiografia; Fraturas do úmero/cirurgia; Fraturas do ombro/radiografia; Fraturas do ombro/cirurgia; Técnicas de sutura; Fixação de fratura

\section{ABSTRACT}

Objective: To evaluate the clinical and radiographic results of patients with two-and threepart fractures of the proximal end of the humerus treated with non-absorbable sutures and with incorporation of the rotator cuff, which allows for increased stability of the fixation, mainly in patients with low bone quality. Methods: 19 patients were operated on, fifteen female and four male, with a mean age of 57.4 years (23-79 years) and a mean follow-up of 53.4 months (seven to 144 months). Based on Neer classification, the authors found ten three-part fractures (surgical neck and larger tuberosity), and nine two-part fractures (two of the larger tuberosity and seven of the surgical neck). All of them were operated in the beach chair position with endotracheal anesthesia and brachial plexus blockade. Deltopectoral approach was used for twopart fractures of the surgical neck and for three-part fractures. For two-part fractures of the larger tuberosity, transacromial approach was preferred. The fractures were reduced and fixated with nonabsorbable number five sutures. The cervicodiaphyseal was measured to evaluate vicious consolidation. Results: The authors checked bone consolidation in $95 \%$ of the patients. The mean anterior elevation in patients with two-part fractures was $163.3^{\circ}\left(90^{\circ}-180^{\circ}\right)$, mean external rotation was $56^{\circ}\left(30^{\circ}\right.$ to $\left.90^{\circ}\right)$, and the mean internal rotation of T10 (S1-T5). In patients with three-part fractures, mean anterior elevation was of $163^{\circ}\left(100^{\circ}-180^{\circ}\right)$, mean external rotation was $52.5^{\circ}$ $\left(5^{\circ}-70^{\circ}\right)$, and the mean internal rotation of T10 ( L4T7). The abduction strength was equal to that of the contralateral side in twelve patients and lesser in 7 patients. The time required for the patient to go back to prior activities was a mean of 5.19 months (two to 12 months). In the radiological evaluation, the mean cervicodiaphyseal angle was of $141^{\circ}\left(110^{\circ}-170^{\circ}\right)$ in two-part fractures, and it was of $150^{\circ}\left(106^{\circ}-210^{\circ}\right)$ in three-part fractures, valgus consolidation being seen in nine patients (59\%), and varus consolidation in two patients. Based on the UCLA score, there were $88.8 \%$ good and excellent results, and $11.2 \%$ of poor results for both types of fracture. The bad results were represented by a case of pseudoarthrosis and a case of adhesive capsulitis in the postoperative phase. Conclusion: Fractures in two- and threeparts of the proximal humerus may be satisfactorily treated with non-absorbable suture incorporating the rotator cuff, specially in the elderly. Metalrelated complications are ruled out. Patients with fractures of the larger tuberosity treated with sutures may have similar results to those with surgical neck fractures. Although the objective of the surgery is anatomic reconstruction, some residual deformity does not prevent a satisfactory result. The method is little invasive, allows for a reasonable fragment stability, with high rates of bone consolidation and patient satisfaction. Patient cooperation is a key factor for the therapeutic success.

Keywords - Humeral fractures/radiography; Humeral fractures/surgery; Shoulder fractures/ radiography; Shoulder fractures/surgery; Suture techniques; Fracture fixation 


\section{INTRODUÇÃO}

Fraturas do úmero proximal correspondem ao terceiro tipo de fratura mais comum vista no idoso e a $10 \%$ de todas as fraturas naqueles com mais de 65 anos, atingindo ossos com má qualidade e sendo duas vezes mais freqüentes nas mulheres ${ }^{(1-6)}$. São, em sua maioria, pouco desviadas e respondem satisfatoriamente à imobilização seguida de movimentação precoce ${ }^{(2)}$. O tratamento cirúrgico é reservado para as fraturas instáveis, que, por ação muscular, produzem uma deformidade rotacional de difícil redução e manutenção.

Inúmeras técnicas são descritas na literatura, incluindo a fixação externa, placas e parafusos ${ }^{(7-15)}$, hastes intramedulares $^{(3,16-17)}$ e hemiartroplastias ${ }^{(6,15,18-19)}$. Resultados desfavoráveis têm sido atribuídos à osteopenia, às grandes desperiostizações necessárias para a colocação das placas e também às migrações, quebras, penetração neurovascular dos pinos percutâneos e reoperação para remoção dos fios ${ }^{(7,10)}$. Atualmente, tem crescido o interesse por técnicas menos invasivas, de menor agressão às partes moles e à vascularização, com conseqüente prejuízo à consolidação óssea, dentre as quais se incluem a redução aberta e fixação por osteossutura, particularmente importante no osso osteopênico ${ }^{(14,20-23)}$.

O objetivo deste trabalho é avaliar os resultados clínicos e radiográficos de pacientes com fraturas em duas e três partes do úmero proximal tratados por redução seguida de estabilização por sutura não absorvível, com incorporação do manguito rotador.

\section{MÉTODOS}

Avaliamos 19 pacientes portadores de fraturas em duas e três partes do úmero proximal ocorridas entre julho de 1993 e dezembro de 2004, fixadas por sutura não absorvível, com incorporação do manguito rotador, avaliados nos Hospitais Governador Israel Pinheiro, Mater Dei e Universitário São José, de Belo Horizonte/MG. Entre os pacientes, 15 eram do sexo feminino e quatro do masculino, com média de idade de 57,4 anos (23-79 anos) e seguimento médio de 53,4 meses (sete a 144 meses). O mecanismo da fratura foi queda da própria altura em 13 pacientes, acidente automobilístico em dois, convulsão em um e, nos de- mais, causas variadas. Os pacientes foram avaliados por radiografias em ântero-posterior (AP) verdadeiro, perfil da escápula e axilar, sendo a última realizada no centro cirúrgico já com o paciente anestesiado. De acordo com a classificação de $\mathrm{Neer}^{(2)}$, encontramos 10 fraturas em três partes - colo cirúrgico (CC) e tubérculo maior (TM) - e nove fraturas em duas partes: duas TM e sete CC (tabela 1); de todas essas fraturas, apenas em uma ocorreu associação com luxação glenoumeral anterior. Exame neurovascular da extremidade fraturada foi realizado em todos os $\operatorname{casos}^{(21,24)}$. Foram pesquisados o tempo e o número de sessões na reabilitação necessárias ao retorno às atividades prévias à fratura. $\mathrm{Na}$ avaliação clínica, foram medidos os graus de rotação externa, rotação interna e elevação anterior. Foi também avaliada a força de abdução, comparando-se o lado afetado com o lado normal, sendo classificada como menor ou igual. Na avaliação radiológica, investigamos a presença de encurtamento, osteólise, necrose avascular, pseudartrose, consolidação viciosa e medimos o ângulo cervicodiafisário; aceitamos como normal o de $130^{\circ}$, considerada como padrão uma linha perpendicular ao colo anatômico e a outra paralela à diáfise umeral ${ }^{(22)}$. Para a avaliação funcional, foi utilizado o escore da Universidade da Califórnia em Los Angeles (UCLA).

\section{Técnica cirúrgica}

Todos os pacientes foram operados na posição em "cadeira de praia", com bloqueio do plexo braquial e anestesia endotraqueal, associação que permite anestesia mais leve e ausência de dor ao acordar, prevenindo, dessa maneira, um movimento descontrolado do paciente que poderia colocar em risco a osteossíntese. $\mathrm{O}$ acesso deltopeitoral foi o preferido para fraturas em duas partes do colo cirúrgico e para as fraturas em três partes $^{(7,24)}$. Para as fraturas em duas partes do tubérculo maior foi utilizado o acesso transacromial, na junção das porções anterior e média do deltóide ${ }^{(23)}$. O deltóide foi dividido ao longo de suas fibras até uma distância não mais que $5 \mathrm{~cm}$ do acrômio, para não pôr em risco o nervo axilar. Após dissecção de partes moles e visualização da fratura, era realizada a curetagem do foco da fratura, deixando-o livre de qualquer 
TABELA 1

Relação dos pacientes

\begin{tabular}{|c|c|c|c|c|c|c|c|c|c|c|}
\hline Nome & Sexo & $\begin{array}{l}\text { Idade } \\
\text { (anos) }\end{array}$ & $\begin{array}{l}\text { Follow-up } \\
\text { (meses) }\end{array}$ & $\begin{array}{l}\text { Class. } \\
\text { (Neer) }\end{array}$ & UCLA & $\mathrm{EA}^{0}$ & $\mathbf{R E}^{0}$ & $\begin{array}{c}\mathbf{R I}^{\mathbf{0}} \\
\text { níveis }\end{array}$ & Radiog. & $\mathrm{ACD}^{0}$ \\
\hline EAG & $\mathrm{M}$ & 45 & 132 & 2P/TM & 29 & 160 & 60 & T8 & A & 132 \\
\hline AGS & $\mathrm{M}$ & 47 & 12 & $3 P$ & 32 & 160 & 60 & $\mathrm{~T} 1$ & $A$ & 130 \\
\hline $\mathrm{ACF}$ & $\mathrm{F}$ & 70 & 29 & $2 \mathrm{P} / \mathrm{CC}$ & 35 & 170 & 50 & T8 & VG & 170 \\
\hline MIS & $\mathrm{F}$ & 55 & 84 & 2P/TM & 35 & 180 & 60 & $\mathrm{T7}$ & A & 145 \\
\hline DR & $\mathrm{F}$ & 67 & 60 & $3 P$ & 28 & 170 & 45 & $\mathrm{T7}$ & VG & 170 \\
\hline MCS & $F$ & 73 & 48 & $3 P$ & 32 & 160 & 45 & L4 & $A$ & 135 \\
\hline TA & $\mathrm{F}$ & 70 & 10 & $3 P$ & 33 & 150 & 70 & T10 & $A$ & 135 \\
\hline AGA & $\mathrm{F}$ & 60 & 27 & $3 P$ & 34 & 180 & 60 & $\mathrm{T7}$ & $\begin{array}{c}\text { VG/TM } \\
\text { ALTA }\end{array}$ & 146 \\
\hline MLS & $\mathrm{F}$ & 79 & 102 & $3 P$ & 35 & 180 & 60 & $\mathrm{T7}$ & $\begin{array}{l}\text { VG/TM } \\
\text { BAIXA/ } \\
\text { OST.TM }\end{array}$ & 168 \\
\hline AMC & $\mathrm{F}$ & 70 & 28 & $3 P$ & 33 & 180 & 80 & T8 & VG & 160 \\
\hline RAM & $\mathrm{F}$ & 38 & 13 & $3 P$ & 18 & 100 & 5 & $\mathrm{~T} 7$ & $\begin{array}{c}\text { VG/TM } \\
\text { ALTA/CA } \\
\text { PÓS-OP }\end{array}$ & 210 \\
\hline HFF & $\mathrm{F}$ & 36 & 40 & $2 \mathrm{P} / \mathrm{CC}$ & 33 & 180 & 90 & T5 & VR & 110 \\
\hline GPC & $F$ & 23 & 111 & $2 \mathrm{P} / \mathrm{CC}$ & 35 & 180 & 50 & T5 & VR & 110 \\
\hline JMF & $\mathrm{F}$ & 60 & 24 & $2 \mathrm{P} / \mathrm{CC}$ & 8 & 90 & 30 & $\mathrm{~S} 1$ & PA & - \\
\hline AMF & $\mathrm{F}$ & 62 & 54 & $2 \mathrm{P} / \mathrm{CC}$ & 29 & 150 & 45 & $\mathrm{T7}$ & $\begin{array}{l}\text { VG/CA } \\
\text { PÓS-OP }\end{array}$ & - \\
\hline ZPF & $\mathrm{F}$ & 52 & 12 & $2 \mathrm{P} / \mathrm{CC}$ & 34 & 180 & 60 & T8 & A & 124 \\
\hline MLM & $\mathrm{F}$ & 77 & 7 & $3 P$ & 34 & 180 & 60 & Т8 & A & 124 \\
\hline NLD & $\mathrm{F}$ & 64 & 78 & $3 P$ & 35 & 180 & 60 & $\mathrm{T7}$ & $\begin{array}{c}\text { VG/OST. } \\
\text { TM }\end{array}$ & 124 \\
\hline JRC & $M$ & 46 & 144 & $2 \mathrm{P} / \mathrm{CC}$ & 34 & 180 & 60 & Т8 & $A$ & 124 \\
\hline
\end{tabular}

Fonte: Hospital Governador Israel Pinheiro/Hospital Mater-Dei/Hospital Universitário São José - Belo Horizonte/MG.

Class. = Classificação; UCLA = Universidade da Califórnia Los Angeles; $E A=$ Elevação anterior; $R E=$ Rotação externa; $R \mathrm{I}=\mathrm{Rotação}$ interna; Radiog. $=$ Achados radiográficos; $A C D=$ Ângulo cervicodiafisário; $M=$ Masculino; $F=$ Feminino; $L A=$ Luxação anterior; $2 P$ $=$ duas partes; $\mathrm{TM}=$ Tuberosidade maior; $3 \mathrm{P}=$ Três partes; $\mathrm{CC}=$ Colo cirúrgico; $\mathrm{D}=$ Direito; $\mathrm{E}=$ Esquerdo; $\mathrm{NR}=\mathrm{Não}$ retornou; $\mathrm{A}=$ Alinhamento anatômico; VG = Valgo; OST.TM = Osteólise da tuberosidade maior; CA PÓS-OP = Capsulite adesiva no pós-operatório; $\mathrm{PA}=$ Pseudartrose $\mathrm{VR}=$ Varo.

interposição de tecidos. Posteriormente, o fragmento distal era preparado com a realização de perfurações com broca de $2,7 \mathrm{~mm}$; nos orifícios confeccionados, eram passados os fios de sutura. Em seguida, a cabeça do úmero era impactada na metáfise, tracionando-se o tubérculo para baixo do nível da superfície articular, obtendo-se posição estável em valgo. Utilizando-se fios inabsorvíveis no 5 de poliéster, passados na junção tendão-osso, era obtida a sutura dos fragmentos em "figura de oito". Testada e confirmada a estabilidade, o braço era imobilizado, em adução-rotação interna, por tipóia. Radiografias semanais eram realizadas para controle da redução e acompanhamento da consolidação (figuras 1A, 1B, 1C). Dependendo do quadro álgico do paciente e da estabilidade obtida no peroperatório, já nos primeiros dias de pós-operatório eram iniciados os movimentos de elevação passiva. A partir da segunda semana, eram acrescentados os exercícios pendulares e, com seis semanas, o paciente era liberado da tipóia para iniciar os exercícios de reforço muscular. 


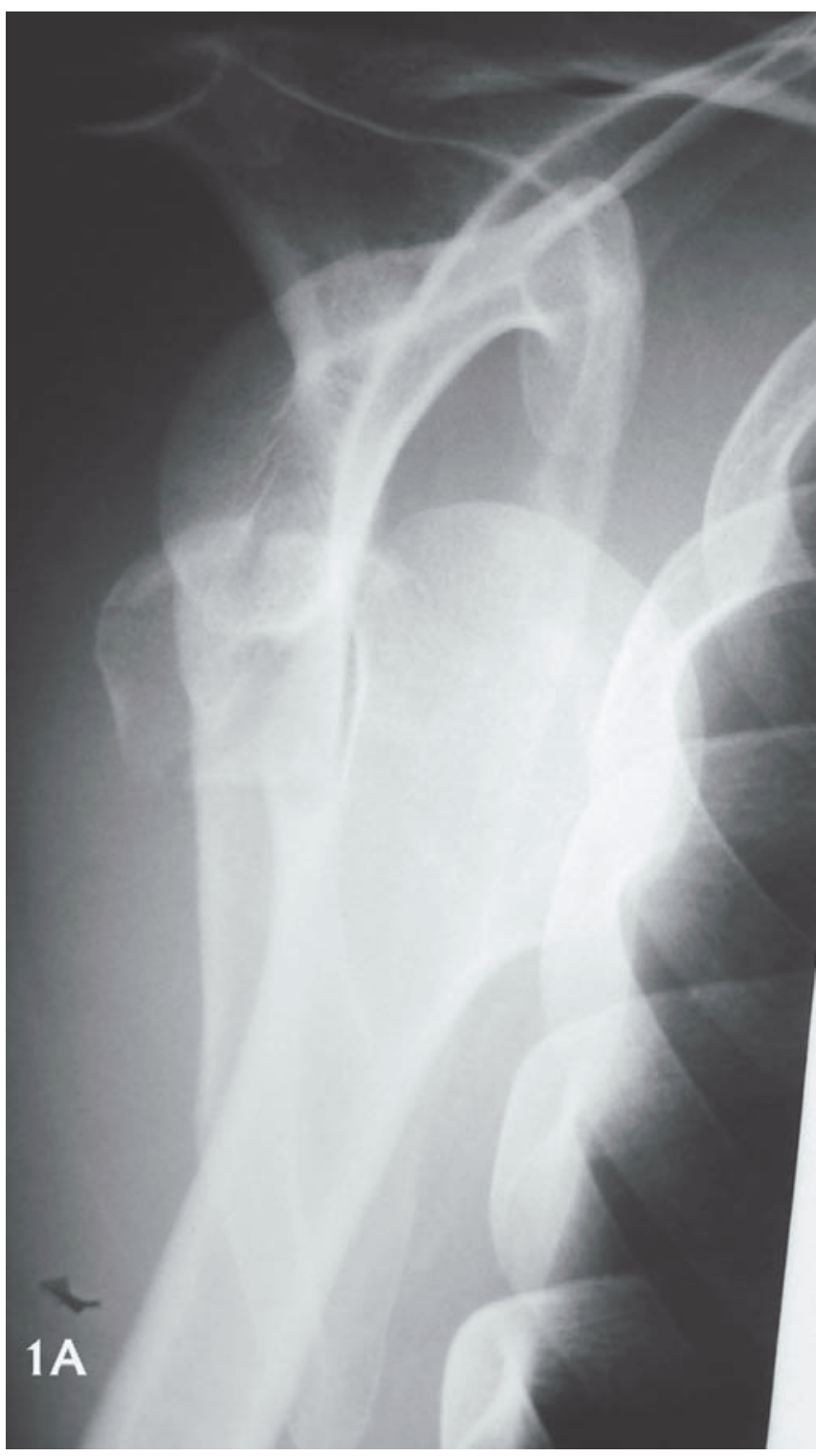

Figura 1A - Radiografia em AP mostrando luxação glenoumeral associada a fratura do tubérculo maior úmero proximal

\section{RESULTADOS}

Em apenas um paciente não houve consolidação óssea. A média da elevação anterior no grupo dos pacientes com fratura em duas partes foi de $163,3^{\circ}\left(90^{\circ}\right.$ $\left.180^{\circ}\right)$; a rotação externa média, de $56^{\circ}\left(5^{\circ}\right.$ a $\left.70^{\circ}\right)$; e a rotação interna média, de T9 (S1-T5) (tabela 1). Nos pacientes com fratura em três partes, a elevação anterior média foi de $141^{\circ}\left(110^{\circ}-170^{\circ}\right)$; a rotação externa

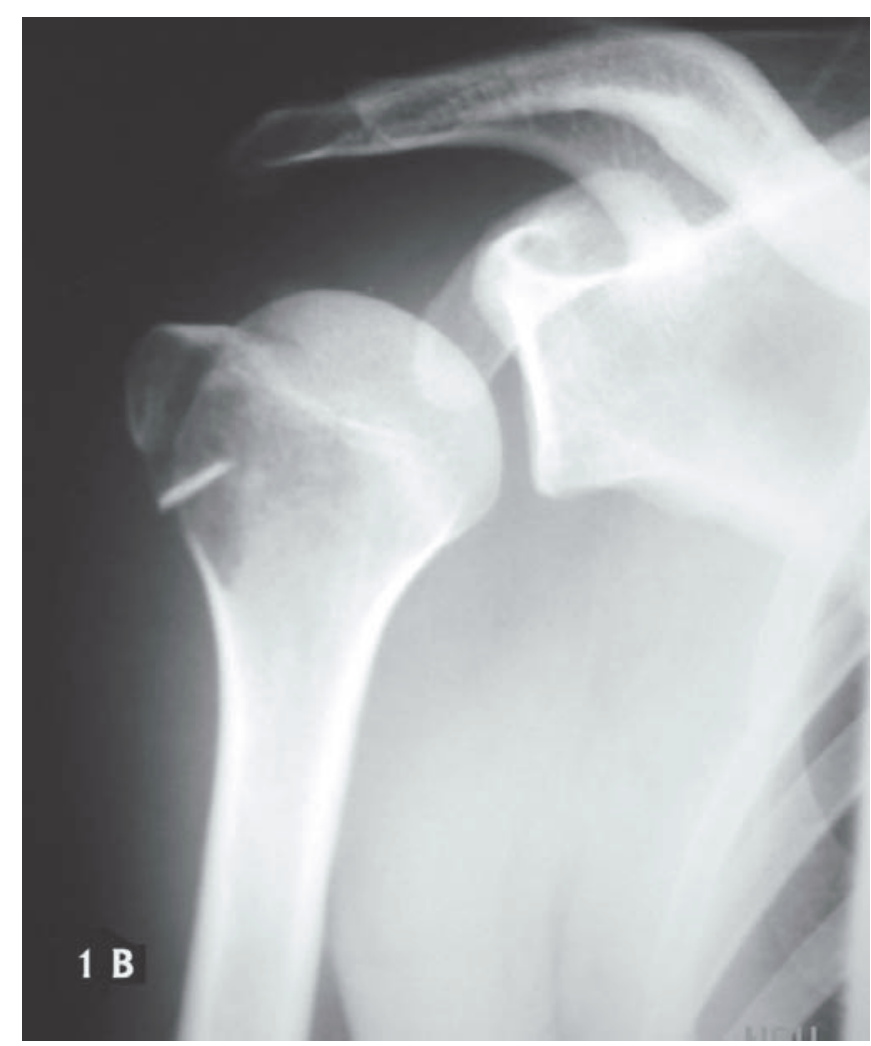

Figura 1B - Redução da luxação, porém com o tubérculo maior não reduzido

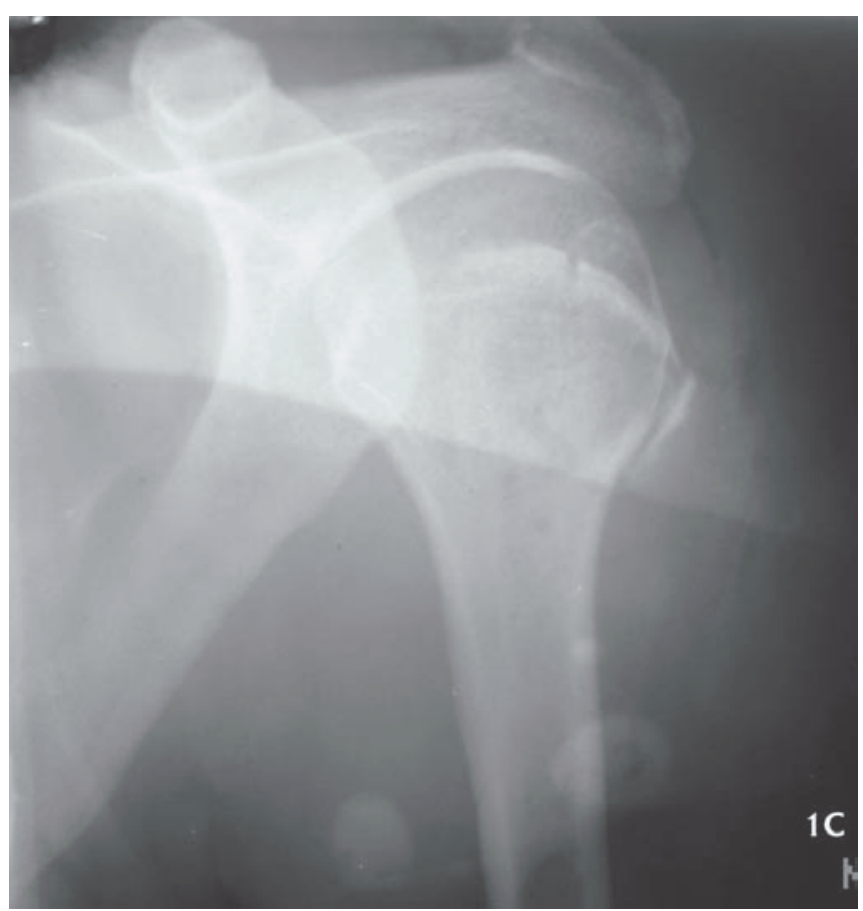

Figura 1C - Controle pós-cirúrgico mostrando boa redução 


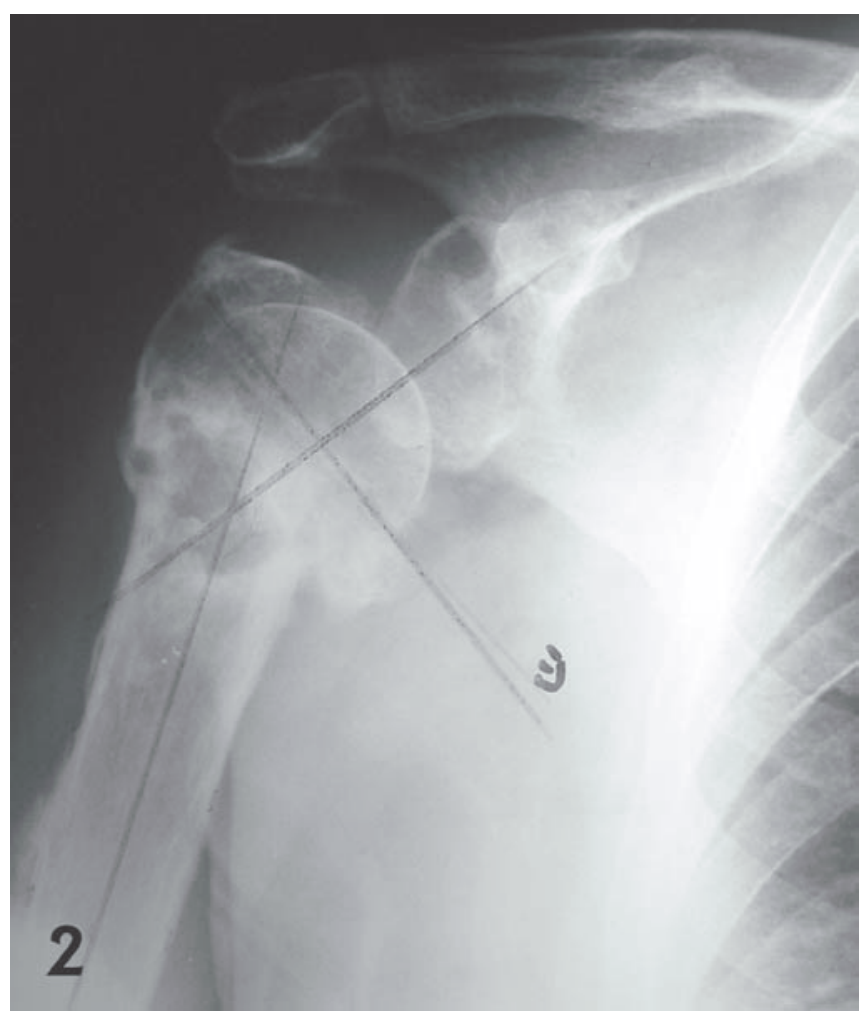

Figura 2 - Paciente de no 11 com consolidação alta do tubérculo maior. UCLA 18.

média, de $52,2^{\circ}\left(5^{\circ}-70^{\circ}\right)$; e rotação interna média, de T10 (L4-T7). A força de abdução foi igual à do lado contralateral em 12 pacientes e menor em sete. O número de sessões de fisioterapia e o tempo necessário para o retorno às atividades prévias foram, respectivamente, 34,8 sessões (duas a 100) e 5,19 meses (dois a 12 meses). Apenas um paciente não retornou às suas atividades. Na avaliação radiológica, o ângulo cervicodiafisário médio foi de $141^{\circ}\left(110^{\circ}-170^{\circ}\right)$ nos pacientes com fratura em duas partes, sendo verificada consolidação em valgo em três pacientes, uma em varo, quatro anatômicas e uma pseudartrose. Nos pacientes com fratura em três partes, o ângulo cervicodiafisário médio foi de $150^{\circ}\left(106^{\circ}-210^{\circ}\right)$, sendo seis consolidações em valgo, uma em varo e três anatômicas (tabela 1). Entre os pacientes, dois tiveram osteólise do tubérculo maior, ambos com fratura em três partes. A consolidação com tubérculo maior alto foi observada em dois pacientes; em um houve consolidação em uma posição mais baixa, todos com fratura em três partes.
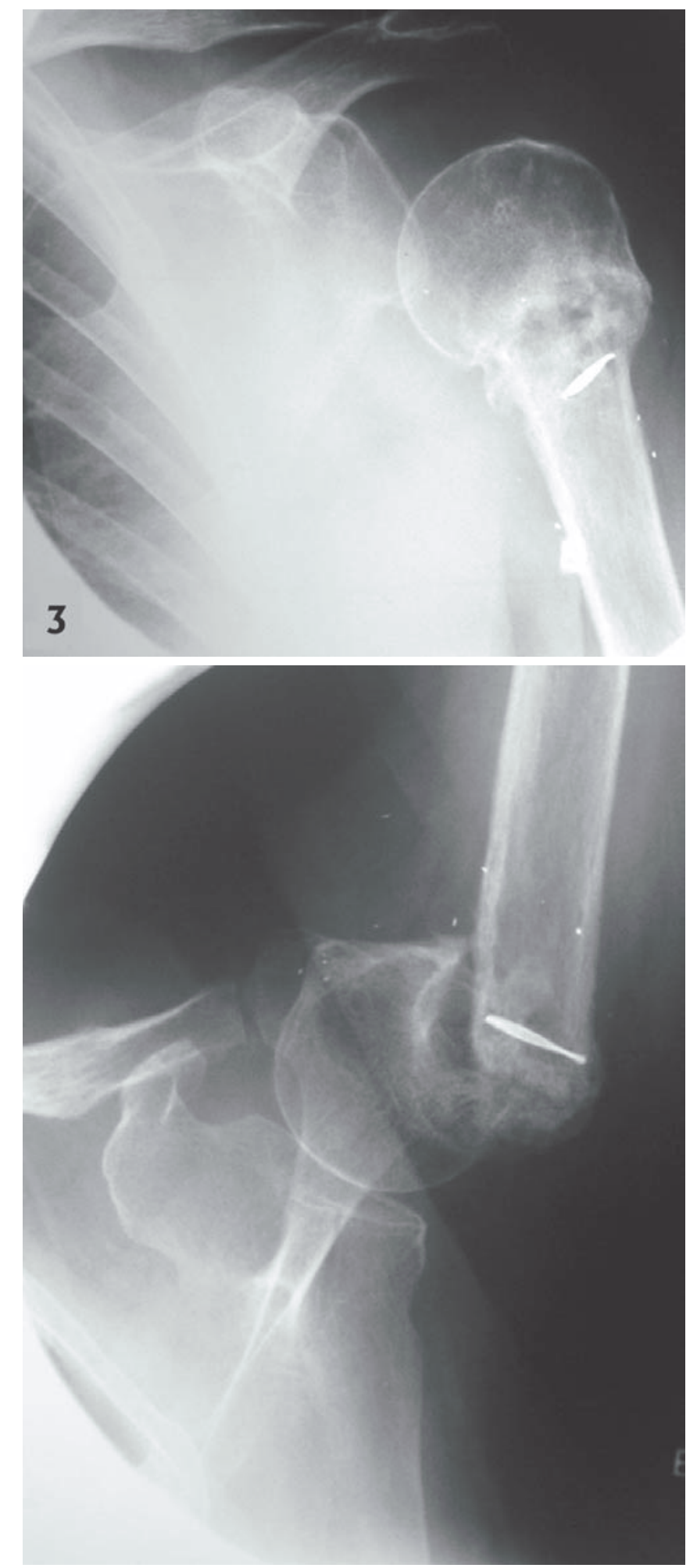

Figura 3 - Radiografia em AP axilar mostrando pseudartrose do úmero proximal, após tentativa de osteossíntese com sutura não absorvível. UCLA 8. 
Em dois pacientes ocorreu capsulite adesiva, um com fratura em duas partes do colo cirúrgico e outro com fratura em três partes. Não foram observadas complicações como necrose avascular, lesão nervosa ou infecção. De acordo com o escore da UCLA, obtivemos $88,8 \%$ de bons e excelentes resultados nos pacientes com fratura em duas partes e os mesmos $88,8 \%$ nos pacientes com fratura em três partes. Os resultados insatisfatórios foram de 11,2\% nos dois grupos. Destes últimos, um paciente evoluiu com pseudartrose do colo cirúrgico (fratura em duas partes) e outro, com quadro de capsulite adesiva com tubérculo maior consolidado em posição alta (fratura em três partes) (figuras 2 e 3 ).

\section{DISCUSSÃO}

As fraturas da extremidade proximal do úmero constituem sério desafio mesmo para os especialistas, devido às situações freqüentemente encontradas, como osteopenia, instabilidade dos fragmentos, presença de co-morbidades e incapacidade - desmotivação ou incoordenação motora de certos pacientes para contribuir com o tratamento ${ }^{(1,3,5-6,12,21-22)}$.

As inúmeras inserções musculares da extremidade proximal do úmero explicam por que é difícil reduzir e manter as fraturas com grande desvio.

O ombro tem muito pouca tolerância aos desvios do tubérculo maior. Segundo Garg et al, McLaughlin sugeriu que desvios maiores que $0,5 \mathrm{~cm}$ poderiam ser problemáticos ${ }^{(25)}$. De acordo com Park et al, desvios maiores do que $0,3 \mathrm{~mm}$ em atletas ou trabalhadores que utilizam o membro superior acima da cabeça deveriam ser reduzidos ${ }^{(22)}$. Em nossa casuística, que envolve média de idade mais alta, toleramos desvios de até $0,5 \mathrm{~cm}$. Nas fraturas envolvendo o colo cirúrgico, angulações maiores do que $45^{\circ}$ não foram aceitas, como recomenda Hoffmeyer ${ }^{(24)}$.

Somos de opinião de que cada paciente deve ter o seu planejamento cirúrgico confeccionado especificamente para ele. Necessário lembrar que o ombro já está seriamente danificado e que qualquer técnica escolhida deve ter como prioridade não traumatizar ainda mais os tecidos. A principal fonte de irrigação da cabeça umeral, o ramo ascendente da artéria circunflexa umeral anterior, passa sob o tendão do bíceps no sulco intertubercular, estando em risco tanto no trauma quanto na dissecção cirúrgica ${ }^{(7,14,21,23-24)}$. A técnica que adotamos proporciona visualização adequada, com agressão mínima às partes moles.

A fixação metálica não é muito bem tolerada em fraturas muito cominutivas ou desviadas em osso osteopênico. Nessas fraturas, o manguito rotador é forte o bastante para segurar a fratura, especialmente nos idosos, e manter todos os fragmentos juntos, criando uma construção estável e, portanto, favorável para a cura da fratura ${ }^{(26)}$. Os benefícios da dissecção mínima necessária para a confecção da osteossíntese com fios não absorvíveis são enormes quando comparados com os riscos de correntes das osteossínteses por placas e parafusos.

A proposta é converter fratura desviada em uma "fratura em uma parte"(22). Flatow et al relataram $82 \%$ de bons e excelentes resultados em 22 pacientes com fraturas em duas e três partes do colo do úmero tratadas por sutura com fios de aço ou sutura com fio inabsorvível ${ }^{(27)}$. Nos casos com cominuição, associaram hastes de Ender para maior estabilidade e mostraram que a fixação por sutura inabsorvível é preferível, porque não necessita retirar o material de síntese ${ }^{(27)}$. Ruch et $a l$, após avaliação biomecânica, justificam a haste intramedular pelo aumento da estabilidade torsional ${ }^{(3)}$. Essa conduta também é confirmada por outros autores $^{(14,16-17)}$. Banco et al descreveram bons resultados em pacientes tratados com sutura com a técnica de "páraquedas" com fios de Dacron $^{\circledR}$ para fraturas em duas partes do colo cirúrgico e chamam atenção para evitar a fixação do tubérculo maior em uma posição mais alta, devido à possibilidade de impacto subacromial ${ }^{(28)}$. Park et al relataram $89,3 \%$ bons e excelentes resultados em fratura em duas e três partes do colo do úmero tratadas por sutura inabsorvível em 28 ombros. Nesse trabalho identificaram alinhamento não anatômico em $14 \%$ dos seus pacientes, fato que não comprometeu o resultado funcional ${ }^{(22)}$.

McLaughlin ponderou que a anatomia normal não é crucial para uma função normal do ombro e que certos graus de desvio são compatíveis com ombro confortável ${ }^{(29)}$. Tal fato foi também verificado nessa pesquisa, pois $47 \%$ dos pacientes tiveram consolidação 


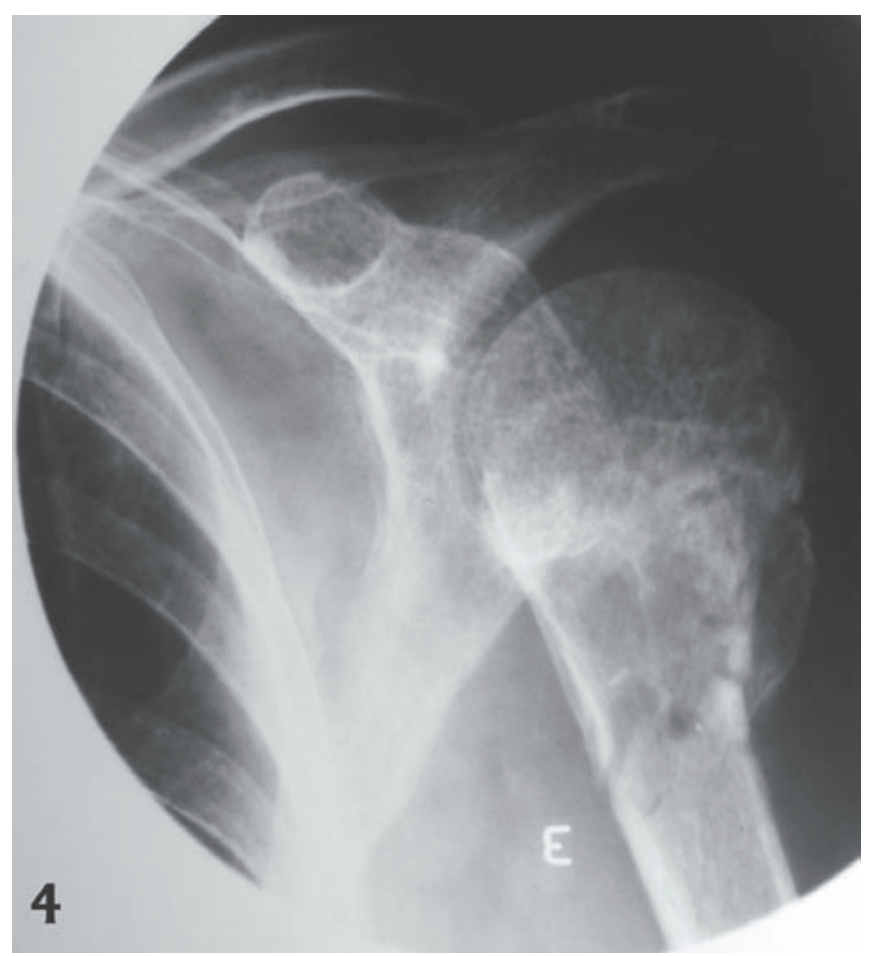

Figura 4 - Radiografia em AP mostrando uma fratura consolidada em valgo. UCLA 35.

em valgo sem prejuízo funcional (figura 4). Não foi possível definir a partir de que grau a deformidade poderia prejudicar a função (figura 5). No ato cirúrgico procuramos criar uma situação estável, impactando-se a metáfise contra a cabeça umeral, mantendo-se um alinhamento do colo cirúrgico até aproximadamente $20^{\circ} \mathrm{em}$ valgo. $\mathrm{O}$ aspecto radiográfico pós-operatório nem sempre agrada aos olhos do cirurgião, mas o importante é a estabilidade da fratura.

Uma limitação do nosso estudo é a medida do ângulo cervicodiafisário, sujeita a erros, por não existirem métodos padronizados para documentar sua reprodução.

Hoffmeyer prioriza o uso de Ethibond ${ }^{\circledR}$ n 6 para a fixação de fraturas da extremidade proximal do úmero em três e quatro partes quando utiliza o método de osteossutura, obtendo adequada estabilidade da fratu$\mathrm{ra}^{(24)}$.

A redução da força de abdução em $40 \%$ dos nossos pacientes não comprometeu os bons resultados. Dos sete pacientes com força diminuída, cinco apresenta-

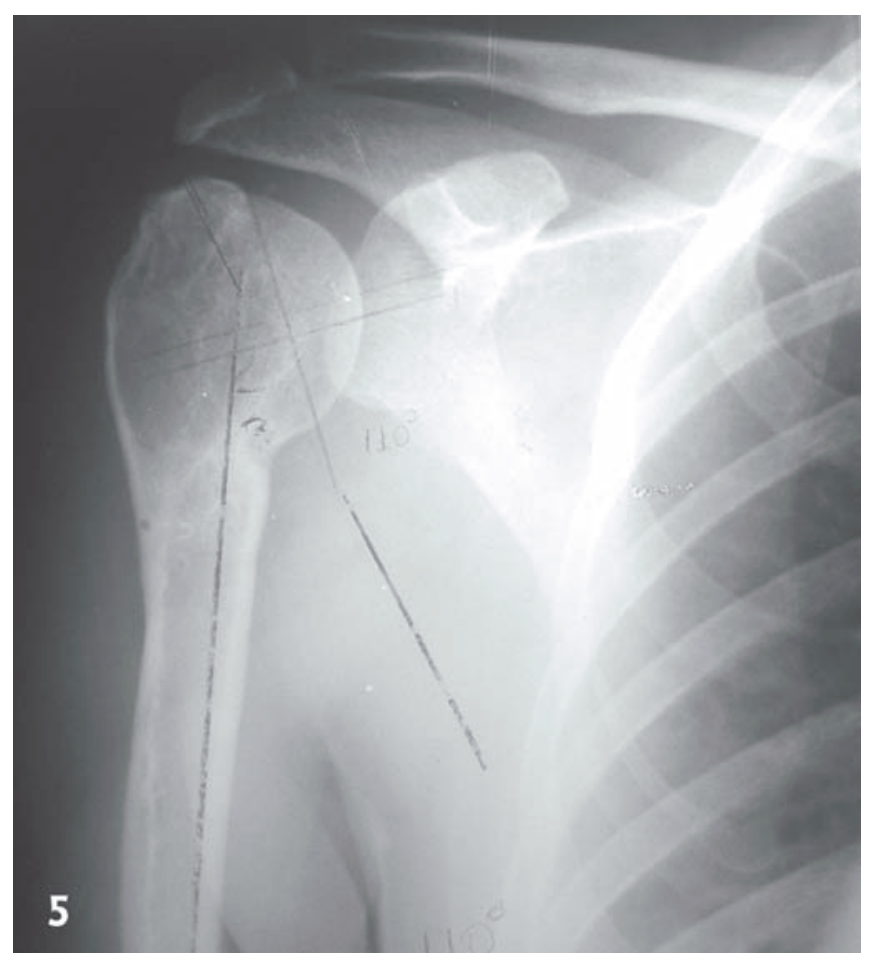

Figura 5 - Consolidação em varo. Bom resultado funcional. UCLA 33.

vam resultados satisfatórios e dois foram incluídos entre os maus resultados; um deles desenvolveu pseudartrose e o outro, capsulite adesiva.

Observamos 10,5\% de casos com osteólise do tubérculo maior e, assim como Park et al ${ }^{(22)}$ observaram em seus casos, não houve prejuízo da função. Em nossos 19 casos também não observamos nenhum paciente com evidência de necrose avascular, provavelmente devido à técnica cirúrgica atraumática e com pouca desperiostização dos fragmentos. Ausência de necrose avascular também foi o achado de Gillespie et al, quando analisaram 24 pacientes com fraturas em duas e três partes do colo do úmero por eles fixadas por osteossutura, na qual acrescentaram sutura lateral para evitar desvio em varo ${ }^{(30)}$. Após o único caso de pseudartrose verificado em nossa casuística, passamos a acrescentar também a sutura lateral por aumentar muito a estabilidade da montagem. Empregando técnica semelhante à que adotamos, Park et al relataram média de elevação anterior, rotação externa e rotação interna, respectivamente, de $155^{\circ}\left(100^{\circ}-175^{\circ}\right), 46^{\circ}\left(15^{\circ}\right.$ - 
$70^{\circ}$ ) e T11 (média contralateral T6) ${ }^{(22)}$. Esses achados equivalem aos do nosso estudo.

A reabilitação pós-operatória responde por grande parte do sucesso terapêutico. Uma vez obtida boa estabilidade no ato cirúrgico e estando o paciente sentindo-se confortável, iniciamos os movimentos passivos. Em virtude de ser essa técnica pouco agressiva às partes moles, permite movimentos pouco dolorosos a partir do segundo ou terceiro dia de pós-operatório, encontrando esta conduta respaldo na literatura pertinente ${ }^{(21,31)}$.

De acordo com o escore da UCLA, registramos $88,8 \%$ de bons e excelentes resultados, índices satisfatórios que demonstram os benefícios da técnica miniinvasiva.

\section{CONCLUSÃO}

O método proposto - redução e osteossíntese com sutura não absorvível - é pouco invasivo, conferindo estabilidade razoável dos fragmentos e altos índices de consolidação, quando respeitados princípios da técnica. A consolidação, em valgo, não leva a prejuízo funcional.

\section{REFERÊNCIAS}

1. Palvanen M, Kannus P, Niemi S, Parkkari J. Update in the epidemiology of proximal humeral fractures. Clin Orthop Relat Res. 2006;442:87-92.

2. Neer CS 2nd. Displaced proximal humeral fractures. I. Classification and evaluation. J Bone Joint Surg Am. 1970; 52(6):1077-89.

3. Ruch DS, Glisson RR, Marr AW, Russell GB, Nunley JA. Fixation of three-part proximal humeral fractures: a biomechanical evaluation. J Orthop Trauma. 2000;14(1):36-40.

4. Chu SP, Kelsey JL, Keegan TH, Sternfeld B, Prill M, Quesenberry CP, Sidney S. Risk factors for proximal humerus fracture. Am J Epidemiol. 2004;160(4):360-7.

5. Olsson C, Petersson CJ. Clinical importance of comorbidity in patients with a proximal humerus fracture. Clin Orthop Relat Res. 2006;442:93-9.

6. Veado MAC, Machado LP, Soares CGN, Souza SVS. Avaliação da função do ombro pós-hemiartroplastias em fraturas em três e quatro partes do úmero proximal. Rev Bras Ortop. 2001;36(5): 161-6.

7. Wijgman AJ, Roolker W, Patt TW, Raaymakers EL, Marti RK. Open reduction and internal fixation of three and four-part fractures of the proximal part of the humerus. J Bone Joint Surg Am. 2002;84-A(11): 1919-25.
8. Checchia SL, Doneux S P, Miyazaki AN, Fregoneze M, Silva LA, Pedro FJ, Alam JAUZ. Resultado do tratamento cirúrgico das fraturas epifisárias da cabeça do úmero. Rev Bras Ortop. 2004;39(8):406-14.

9. Checchia SL, Doneux S P, Miyazaki AN, Fregoneze M, Silva LA, Lobo AC, et al. Avaliação do tratamento cirúrgico da fratura em duas partes do colo cirúrgico do úmero com placa PFS 80® ${ }^{\circledR}$. Rev Bras Ortop. 2004;39(10): 555-67.

10. Meier R, Messmer P, Regazzoni P, Rothfischer W, Gross T. Unexpected high complication rate following internal fixation of unstable proximal humerus fractures with an angled blade plate. J Orthop Trauma. 2006;20(4):253-60.

11. Koukakis A, Apostolou CD, Taneja T, Korres DS, Amini A. Fixation of proximal humerus fractures using the PHILOS plate: early experience. Clin Orthop Relat Res. 2006;442:11520.

12. Pritsch P. Fracture of the proximal humerus - internal fixation surgical treatment (closed pinning \& ORIF-PDS suture). J Bone Joint Surg Br. 2002;84-B Suppl 3:295.

13. Resch H, Povacz P, Fröhlich R, Wambacher M. Percutaneous fixation of three- and four-part fractures of the proximal humerus. J Bone Joint Surg Br. 1997;79(2):295-300.

14. Iannotti JP, Ramsey ML, Williams GR Jr, Warner JJ. Nonprosthetic management of proximal humeral fractures. Instr Course Lect. 2004;53:403-16.

15. Henshaw DR, Murthi AM, Levine WN. Percutaneous pins versus ORIF, or is HHR Better? Curr Opin Orthop. 2001;12(4): 307-14.

16. Ferreira Neto AA, Ferreira Filho AA, Zoppi Filho A, Benegas E, Negri JH, Machado LFMC, Oliveira FR. Osteossíntese das fraturas em duas e três partes da extremidade proximal do úmero com hastes de Ender modificadas associadas com amarrilhos de "Ethibond". Rev Bras Ortop. 1997;32(9):707-12.

17. Mighell MA, Jennings WB, Frankle MA. Technique for unstable two-part surgical neck proximal humeral fractures utilizing an intramedullary staple device: the Evan's staple. Tech Shoulder Elbow Surg. 2003;4(2):84-8.

18. Checchia SL, Doneux S P, Miyazaki AN, Fregoneze M, Silva LA, Faria FN, et al. Tratamento das fraturas do terço proximal do úmero com a prótese parcial Eccentra ${ }^{\circledR}$. Rev Bras Ortop. 2005; 40(3):130-40.

19. Neer CS 2nd. Displaced proximal humeral fractures. II. Treatment of three-part and four-part displacement. J Bone Joint Surg Am. 1970;52(6):1090-103.

20. Choi $\mathrm{CH}$, Cuomo F. Proximal humeral fractures. Curr Opin Orthop. 2000;11(4):256-63.

21. Rees J, Hicks J, Ribbans W. Assessment and management of three- and four-part proximal humeral fractures. Clin Orthop Relat Res. 1998;(353):18-29.

22. Park MC, Murthi AM, Roth NS, Blaine TA, Levine WN, Bigliani LU. Two-part and three-part fractures of the proximal 
humerus treated with suture fixation. J Orthop Trauma. 2003; 17(5):319-25.

23. Green A, Izzi J Jr. Isolated fractures of the greater tuberosity of the proximal humerus. J Shoulder Elbow Surg. 2003;12(6): 641-9.

24. Hoffmeyer P. The operative management of displaced fractures of the proximal humerus. J Bone Joint Surg Br. 2002;84(4): 469-80. Erratum in: J Bone Joint Surg Br. 2002;84(7):1091.

25. Garg A, McQueen MM, Court-Brown CM. Nerve injury after greater tuberosity fracture dislocation. Proceedings of Orthopaedic Trauma Association; Charleston, SC; 1999.

26. Codman EA. Fractures in relation to the subacromial bursa. In Codman EA, editor. The shoulder. Rupture of the supraspinatus tendon and other lesions in or about the subacromial bursa. Boston: Thomas Todd; 1934. p. 313-33.
27. Flatow EL, Cuomo F, Maday MG, Miller SR, McIlveen SJ, Bigliani LU. Open reduction and internal fixation of two-part displaced fractures of the greater tuberosity of the proximal part of the humerus. J Bone Joint Surg Am. 1991;73(8):1213-8.

28. Banco SP, Andrisani D, Ramsey M, Frieman B, Fenlin JM Jr. The parachute technique: valgus impaction osteotomy for twopart fractures of the surgical neck of the humerus. J Bone Joint Surg Am. 2001;83-A Suppl 2(Pt 1):38-42.

29. McLaughlin H. Injuries of the shoulder and arm. In: McLaughlin H, editor. Trauma. Philadelphia: WB Saunders; 1959. p. 233-98.

30. Gillespie G, Dabke H, Roberts P, Kulkarni R. Al suture affords increased stability to suture fixation in 2-3 part proximal humeral fractures. J Bone Joint Surg. 2004;86-B Suppl 1:98.

31. Hodgson S. Proximal humerus fracture rehabilitation. Clin Orthop Relat Res. 2006;442:131-8. Review. 